\title{
Frontières
}

\section{Peindre sa mort et celle des autres}

\section{Danse macabre de Niklaus Manuel Deutsch}

\section{Hans-Jürgen Greif}

Volume 19, numéro 2, printemps 2007

Penser sa mort?

URI : https://id.erudit.org/iderudit/017491ar

DOI : https://doi.org/10.7202/017491ar

Aller au sommaire du numéro

Éditeur(s)

Université du Québec à Montréal

ISSN

1180-3479 (imprimé)

1916-0976 (numérique)

Découvrir la revue

Citer cet article

Greif, H.-J. (2007). Peindre sa mort et celle des autres : Danse macabre de Niklaus Manuel Deutsch. Frontières, 19(2), 12-18.

https://doi.org/10.7202/017491ar
Résumé de l'article

Qu'un peintre représente la mort des autres, voilà rien d'inhabituel, surtout s'il s'agit d'une des nombreuses danses macabres au Nord des Alpes, à la fin du Moyen Âge, et dont la plus célèbre fut sans doute celle du monastère des Innocents à Paris (1424/1425, détruite en 1529; gravures de Guy Marchant en 1485). Mais la présence du peintre dans la suite de ceux que la Mort emmène est bien plus rare. Les questions auxquelles l'article tente de donner une réponse sont les suivantes : pourquoi ce peintre suisse du début du XVI ${ }^{\mathrm{e}}$ siècle s'est-il joint à la danse macabre ? Quelle place occupe ce vado mori dans son oeuvre?
Ce document est protégé par la loi sur le droit d'auteur. L'utilisation des services d'Érudit (y compris la reproduction) est assujettie à sa politique d'utilisation que vous pouvez consulter en ligne.

https://apropos.erudit.org/fr/usagers/politique-dutilisation/ 


\section{Résumé}

Qu'un peintre représente la mort des autres, voilà rien d'inhabituel, surtout s'il s'agit d'une des nombreuses danses macabres au Nord des Alpes, à la fin du Moyen Âge, et dont la plus célèbre fut sans doute celle du monastère des Innocents à Paris (1424/1425, détruite en 1529; gravures de Guy Marchant en 1485). Mais la présence du peintre dans la suite de ceux que la Mort emmène est bien plus rare. Les questions auxquelles l'article tente de donner une réponse sont les suivantes: pourquoi ce peintre suisse du début du XVIe siècle s'est-il joint à la danse macabre? Quelle place occupe ce vado mori dans son œuvre?

Mots clés: danse macabre -

autoreprésentation - identité.

\begin{abstract}
There is nothing unusual in a painter depiciting the death of other people, especially in one of the many "dances of death" produced north of the Alps, in the late Middle Ages; the most famous such work was, without doubt, the one at Paris's Church of the Holy Innocents (1424/25, destroyed 1529; engravings published by Guy Marchant in 1485). It is rather rare, though, for the artist to portray himself as one of the people being taken away by Death. This article tries to find an answer to the following questions: why did this Swiss painter represent himself, at the beginning of the 16 th century, as part of the dance? What is the importance of this imposing cycle and his vado mori within his work?
\end{abstract}

Keywords: Dance of Death Self-representation - Identity.

\section{Peindre sa mort et celle des autres Danse macabre de Niklaus Manuel Deutsch}

\author{
Hans-Jürgen Greif, \\ professeur associé, Département des littératures, \\ Université Laval.
}

Les images de la Mort retirant de ce monde un vivant pour le conduire à l'éternité changent d'une religion à l'autre, d'une civilisation à l'autre, d'une époque à l'autre. Ainsi, la fin du Moyen Âge, par exemple, fut une période particulièrement féconde en représentations de la Mort en peinture: le Jugement Dernier, des gravures sur bois montrant des danses macabres, dont les plus célèbres se trouvaient à Paris, à Bâle, à Strasbourg, sans oublier l'Ars moriendi, un Blockbuch publié à Heidelberg, en $1485^{1}$. Mes recherches récentes sur un tableau de Niklaus Manuel Deutsch (1484-1530), peintre suisse du temps de la Réforme, Le Jugement de Pâris (1517-1518), m'ont amené à analyser une autre œuvre de sa main, la Danse macabre, qui fut dans son temps le monument artistique le plus célèbre de Berne. C'est une série de 46 tableaux comportant un autoportrait du peintre interrompu en plein travail par la Mort. Les panneaux qui m'intéressent montrent les scènes 9,44 et $45^{2}$. Le sujet du premier est la figure du prieur, représentant le clergé et ses excès contre lesquels s'élèvent les fidèles. Le deuxième, qui est en fait constitué d'un panneau double, représente un tableau à peine achevé ainsi que l'autoportrait du peintre.

Les spécialistes de l'œuvre manuelienne donnent des indications convaincantes quant au caractère monumental de l'ensemble. Selon toute vraisemblance, la suite des tableaux s'étendait sur une longueur entre quatre-vingts et cent mètres ; la hauteur de chaque tableau devait atteindre au moins deux mètres. Ce qui signifie que les personnages étaient représentés grandeur nature. Si l'on ajoute les quatrains explicatifs, placés sous les panneaux, le mur de support devait atteindre entre $2,5 \mathrm{~m}$ et $3 \mathrm{~m}$. La série se trouvait à l'intérieur de l'enceinte du cimetière appartenant au monastère des dominicains (aujourd'hui : "L'église française»); aux passants, elle était accessible à tout moment ${ }^{3}$.

Les scènes ont été peintes entre 1516 et 1519. Leur création coïncide précisément avec l'époque où la politique de l'Église, surtout dans le domaine financier, allait provoquer de violents débats, tant parmi la population qu'au sein même du clergé. Afin de mieux comprendre la portée de cette fresque et des textes qui l'accompagnaient, il faut brièvement resituer l'ensemble dans son contexte politique et religieux. 


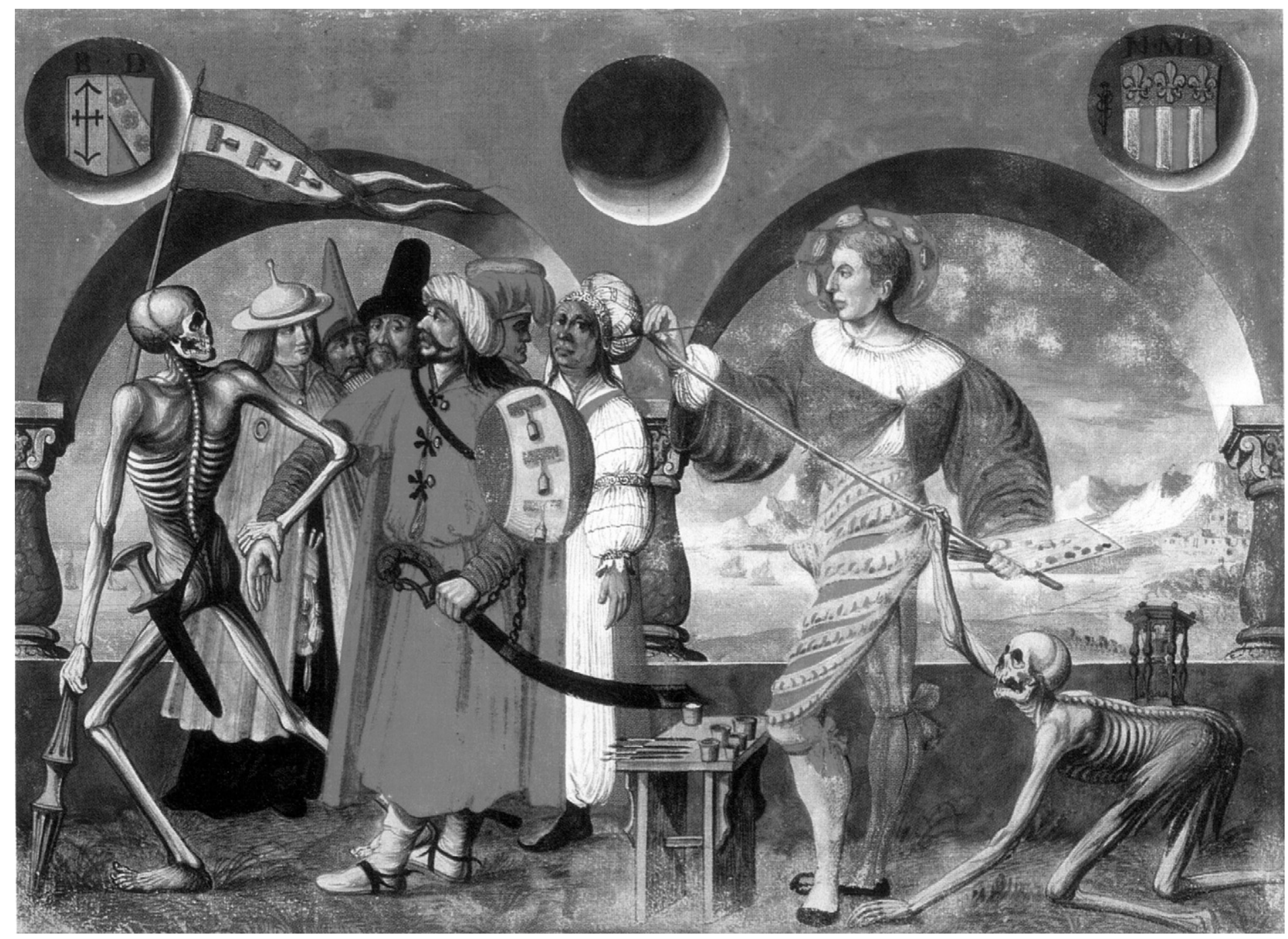

Copie de l'un des panneaux (scènes 44 et 45) de la Danse macabre de Niklaus Manuel Deutsch (1484-1530). Comme la totalité de l'œuvre a été détruite en 1660, il ne nous est parvenu que cette copie à la gouache par Albrecht Kauw (1649). On y voit un autoportrait du peintre interrompu en plein travail par la Mort.

Afin de financer la construction de la basilique de Saint-Pierre, le plus grand édifice du monde occidental au temps de la Renaissance, les papes avaient instauré le système des «lettres d'indulgences». Commencée sous Jules II en 1506, l'entreprise architecturale romaine engloutissait des sommes énormes, levées partout en Europe par les émissaires du pape. Ils parcouraient les pays et encourageaient les fidèles à racheter, par le truchement de «lettres d'indulgences», du temps de pénitence au purgatoire.

Quand Martin Luther, jeune professeur à l'université de Wittenberg, fondée en 1502 par le duc Frédéric le Sage, se rendit compte que ses ouailles préféraient racheter leurs péchés par l'entremise d'un marchand investi de l'autorité papale plutôt que de se rendre au confessional, le moine allemand rédigea 95 thèses devant servir de base à une disputatio entre confrères et qui furent affichées sur les portes de l'église de Wittenberg, le soir du 31 octobre 1517. Ces thèses ne mettaient pas en doute les peines imposées par l'Église; elles se concentraient sur la pertinence des pratiques de Rome permettant au fidèle de racheter ses péchés, même graves, et d'accéder au paradis en payant sur terre son droit d'entrée. Copiées et imprimées à la hâte, les thèses se répandirent à une vitesse vertigineuse partout au nord des Alpes, de la France à la Scandinavie, où les princes leur firent un accueil souvent enthousiaste : ils voyaient d'un mauvais œil la perte de sommes considérables au profit de Rome, où les papes, et tout particulièrement Alexandre VI, Jules II et Léon X, se comportaient comme n'importe quel autre souverain de la Renaissance, c'est-à-dire en politiciens cherchant à étendre leurs aires d'influence et à agrandir la superficie de l'État pontifical. Afin d'augmenter le prestige de leur règne, ces papes furent des mécènes dans chaque domaine de l'art, plus particulièrement en architecture et en peinture. Les thèses luthériennes tombaient au moment précis où le faste et le luxe de Rome faisaient pâlir le souvenir de la plus élégante cour d'Europe, celle de Charles le téméraire, en Bourgogne. La disputatio souhaitée par Luther n'eut jamais lieu. Par contre, ses thèses allaient provoquer le plus grave schisme de l'Église et mener, après le sac de Rome (1527) et quelques deux siècles plus tard, à la perte définitive du pouvoir de l'Église comme puissance séculaire.

Après ce bref aperçu de la situation politique et religieuse en Europe autour de l'an 1517 et avant d'entamer la réflexion sur l'autoreprésentation du peintre dans sa danse macabre, une question s'impose : pourquoi, en pleine Renaissance, un peintre bernois a-t-il accompli une œuvre dont le caractère s'inscrit davantage dans la pensée du Moyen Âge tardif que dans la nouvelle philosophie de la Renaissance? 
Surtout, s'il a été plus d'une fois en contact avec les idées et les œuvres italiennes lors de sa participation aux guerres que se livraient devant Milan, les papes, les empereurs Frédéric III et Maximilien I ${ }^{\mathrm{er}}$, les rois Louis XII et François I ${ }^{\text {er }}$ ? Avec son appropriation des œuvres artistiques de l'Antiquité gréco-romaine, avec le rejet des formes de l'ère gothique, tant en architecture que dans les arts plastiques, par la fondation d'écoles de pensées, comme le platonisme de l'Académie de Florence ou encore l'aristotélisme de l'université de Padoue, l'influence de la Renaissance italienne s'étendait dans tous les pays européens. Cette influence se manifeste clairement dans les arches que nous retrouvons dans cette Danse, dans les paysages idéalisés, dans les effets de perspective, sans oublier la composition des panneaux du peintre. Pourtant, dans la représentation des personnages ainsi que des squelettes, la manière dont la Mort empoigne les vivants, jusqu'au ton rude, parfois grossier des quatrains, Niklaus Manuel se situe clairement dans la tradition du Moyen Âge tardif.

Pour commencer, il faut dire que nous ne pouvons que spéculer sur les années d'apprentissage de ce peintre. Par ailleurs, les chercheurs s'accordent pour dire que, lors des campagnes des mercenaires suisses en Italie (le «Reyslouffen», terme auquel je reviendrai), Niklaus Manuel ait eu l'occasion ou encore le loisir de se familiariser avec la nouvelle peinture italienne. Selon toute vraisemblance, Manuel a appris son métier dans un des ateliers bernois spécialisés dans la fabrication de vitraux ${ }^{4}$. Pendant ses années de compagnonnage, il a vraisemblablement parcouru les régions méridionales de l'Empire et vu des œuvres importantes des Dürer, Schongauer, Nithart (aussi connu sous le nom de Mathias Grünewald) ou les tableaux des deux Holbein. Il aura sans doute étudié des Danses macabres, encore en vogue à ce moment-là, et certainement celle de Bâle. La composition de son œuvre trahit la connaissance des gravures lyonnaises de Mathieu Husz (Tripps, 2005, p. 12). Par conséquent - ses dessins, ses retables, ses deux versions du martyre de saint Jean le prouvent hors de tout doute -, il était encore fortement ancré dans la tradition de l'art gothique. Ses nus féminins, comme le montre le traitement de Vénus et de Pallas dans son Jugement de Pâris, suivent fidèlement ceux de Dürer, à tel point que certains chercheurs ont avancé la thèse que Manuel en a été l'élève.

À cette époque, au nord des Alpes, le peintre est considéré comme un artisan, au même titre qu'un orfèvre, un forgeron, un cordonnier ou un tonnelier, artisan qui, même après plusieurs années d'appren- tissage et de compagnonnage, ne signe que rarement son travail ${ }^{5}$. Son métier exige une bonne observation de la nature tout comme du corps humain, la faculté de saisir les mouvements du corps et des vêtements, de les fixer rapidement sur un support, la connaissance des différentes techniques de peinture (al fresco, maigre ou grasse, a tempera, à l'huile) ainsi que des pigments et de leur réactivité les uns envers les autres. Comme n'importe quel artisan, un peintre vit de commandes. Pour se faire connaître, pour s'imposer aussi face à une concurrence importante, il doit disposer de travaux exposés et vus par le plus grand nombre de personnes, comme les retables dans les églises, les fresques sur les murs d'un édifice public. les commandes les plus nombreuses de la ville, et qui allaient l'engager dans la politique du mouvement de la Réforme, dont il allait devenir une des figures importantes à l'intérieur de la Confédération.

À sa naissance, Manuel s'appelle Niklaus Emmanuel Alleman, ou, plus vraisemblablement, de Alamanis ${ }^{6}$. En 1509, peu après son retour à Berne, et après ses années de compagnonnage, il épouse Katharina Frisching, fille d'un homme riche, bailli au château d'Erlach, territoire sous juridiction bernoise. La mère du peintre, Margaretha Fricker, est issue de la petite bourgeoisie. Le père, qui donne son nom à cet enfant né hors mariage, est apothicaire, lui-même fils d'un apothicaire immigré de Chieri, bourgade située non loin de Turin. Dès qu'il devient

UNE GRANDE CEUVRE, EXPOSÉE COMME AUTANT D'AFFICHES DE PUBLICITÉ DANS UN ENDROIT PARTICULIÈREMENT PASSANT,

PARCE QUE PROCHE D'UNE DES RUES LES PLUS IMPORTANTES DE LA VILLE, ÉTAIT FORTEMENT REMARQUÉE ET DISCUTÉE

TANT PAR LA COMMUNAUTÉ LOCALE QUE PAR LES VOYAGEURS.

La Danse macabre sur le mur du cimetière des dominicains répond précisément à l'exigence de se faire connaître, davantage encore que les retables exécutés pour les églises. Fermés la plupart du temps, ils révèlent les panneaux intérieurs, souvent plus spectaculaires que ceux de l'extérieur, seulement le dimanche et les jours de fête. Une grande œuvre, exposée comme autant d'affiches de publicité dans un endroit particulièrement passant, parce que proche d'une des rues les plus importantes de la ville, était fortement remarquée et discutée tant par la communauté locale que par les voyageurs qui en parlaient chez eux dès leur retour de Berne.

Ce qui ajoutait du poids à cette œuvre "promotionnelle», pour employer un terme plus moderne, c'était l'ensemble des histoires rattachées aux scènes, toutes des commandes issues de la meilleure bourgeoisie de la ville-État, donc des familles les plus fortunées qui voyaient là une belle occasion de laisser leur souvenir aux générations à venir sous forme de portrait d'un de leurs membres, tout comme par leurs armoiries, témoins de leur importance au sein de la ville.

Cependant, la réalisation de la Danse n'aurait sans doute pas eu lieu si la vie du jeune peintre, revenu depuis peu à Berne, n'avait pas connu des changements importants qui devaient en faire le peintre avec membre d'une famille influente, le jeune homme change de nom. Il germanise celui du père, qui devient "Deutsch", enlève de son deuxième prénom la première syllabe, signe désormais tous ses travaux «NMD», longtemps suivi d'un «V.B.» («von Bern») et ajoute la dague suisse ainsi que plusieurs jolies boucles. Il me semble évident que par cette nouvelle identité, le peintre voulait prouver d'abord à la ville, ensuite aux autres membres de la Confédération, qu'il était un Suisse véritable. Il devait faire disparâ̂tre ses origines de "Walch» (aujourd'hui : «Welsch»), signifiant roman, italien ou français et, par extension, étranger, et se présenter comme un homme de souche bernoise ou, du moins, suisse alémanique, les étrangers demeurant toujours suspects dans une société hiérarchisée au plus haut degré, méfiante devant tout ce qui ne tombait pas dans les catégories du connu et du familier.

Ainsi, l'avenir politique se dessine dès le début de sa carrière d'artisan à Berne. L'année suivant son mariage, Niklaus Manuel Deutsch est élu membre du Grand Conseil de Berne, preuve éloquente de l'influence exercée par sa belle-famille. Sans en avoir la certitude, je demeure persuadé que les commandes pour la Danse ont été rendues plus faciles à cause des relations entretenues par sa femme avec les familles importantes, capables de 
débourser les sommes nécessaires pour les tableaux. Dès 1513, le peintre travaille sans relâche. Il confectionne une série de retables, achète un an plus tard une maison dans la belle rue de la Justice (le numéro 72 de la Gerechtigkeitsgasse). Pour mousser encore davantage les affaires, il propose de peindre sa Danse. Et voilà qu'on se presse pour avoir sa place dans ce memento mori public. En effet, nous pouvons identifier, à quelques exceptions près, les commanditaires de la Danse: il s'agit d'une cinquantaine de membres de la grande bourgeoisie, dont Hans Frisching, le beau-frère de Manuel.

Dans ces tableaux, la société bernoise, conservatrice (et donc fortement liée aux traditions du Moyen Âge), consciente de sa valeur, libre dans ses décisions - la ville est membre de la Confédération depuis 1353 se prête volontiers au jeu de la Mort tel qu'interprété ailleurs, mais dans des costumes à la mode, au début du XVI ${ }^{\mathrm{e}}$ siècle. Comme c'est le cas pour d'autres danses macabres, personne ne manque à l'appel. Dans treize tableaux, la Mort emmène d'abord les représentants de l'Église, du pape à la béguine, en passant par le cardinal, le prieur, parmi d'autres. Ensuite, c'est le tour de 28 personnages du monde laïque, de l'empereur au chevalier, du bailli au marchand, sans oublier l'artisan, le mendiant, le cuisinier ou le paysan.

Quand on lit les quatrains qui accompagnent les tableaux, il devient évident que Niklaus Manuel se faisait le porte-parole de l'opinion de la classe dirigeante, les Burger, particulièrement en ce qui concerne le comportement des prêtres. La violence des propos, le manque de respect envers les dignitaires, le langage cru, tout traduit le désenchantement, le dégoût, l'extrême impatience du fidèle envers une institution qui, à cause des scandales à répétition, avait perdu sa crédibilité. Prenons comme exemple la façon dont la Mort s'adresse au prieur:

[La Mort]
Monsieur le prieur,
vous êtes bien gros et gras,
Sautez avec moi dans ce cercle!
Mais vous êtes baigné
de sueurs froides!
Fi, fi donc, vous lâchez de gros étrons!

[Le prieur]

Les friandises m'ont fait grand bien, Dans mes mains, j'ai tenu des fortunes,

Je les ai utilisées pour la luxure

de mon corps,

Qui sera désormais profané

par les vers?.

(Texte de la copie de Kauw; traduction de l'auteur.)
Le ton adopté par la Mort est pour le moins irrévérencieux. Mais la «confession » du prieur - à partir de maintenant, il n'est plus nécessaire de maintenir un semblant de dignité, ni de feindre une vie respectable et conforme avec les règles de son ordre - est exactement celle qu'attendent les fidèles qui connaissent bien le luxe des monastères, les mœurs très libres des moines et des religieuses, la richesse ostentatoire des églises et des chapelles, l'augmentation quotidienne de cette même richesse par des donations, des legs, obtenus souvent par la ruse ou la peur du mourant devant les flammes de l'enfer. Il est frappant de constater que la réplique du prieur n'exprime aucun repentir quant à sa vie passée, il n'a que du regret de quitter la terre au moment où l'existence est si agréable et l'horreur de savoir que son corps, qui lui a procuré tant de plaisirs, pourrira avant le temps, car il est jeune encore.

Ici, Manuel vise un double but. D'abord, il exprime l'irritation des fidèles envers le comportement du clergé, se rangeant ouvertement du côté des opposants qui réclament un changement radical des pratiques religieuses. Autrement dit, il fait ouvertement de la politique. D'un autre côté, il se présente comme un écrivain dont les vers, faciles à retenir, contribuent à mousser constamment le mécontentement des passants, qui, même s'ils ne savent pas lire, connaissent le contenu des quatrains. Stratégie ingénieuse qui portera fruit dès que l'Église catholique sera remplacée par l’Église réformée. Avec la disparition du clergé comme employeur principal des peintres, bon nombre d'entre eux doivent changer de métier ou œuvrer dans un domaine annexe, comme la sculpture ou la fabrication, toujours très à la mode, de vitraux pour les riches bourgeois. D'autres, comme Hans Holbein, s'exilent, en Angleterre ou dans les pays scandinaves ${ }^{8}$.

Que Manuel ait fait précéder son autoportrait par une représentation du fou n'est sans doute pas un hasard, car ce personnage englobe tous les autres, comme l'ont bien démontré Sébastien Brant dans sa Nef des fous (Das Narrenschiff, 1494) ou encore Érasme dans sa Laus stultitiae (1511). En même temps, le fou exprime la vanitas: la Mort détruit ce qu'a créé l'ambition de l'homme; rien ni personne ne résiste au temps. En somme, le fou traduit le sentiment du peintre et de la nouvelle philosophie: qu'il faut prouver sa valeur tant qu'on est en vie et s'ancrer dans le présent puisque l'avenir est incertain.

Plus surprenant est le sujet sous le pinceau de Manuel au moment de sa mort. Il s'agit d'un groupe de six personnages, des
Juifs, un Turc et une Turque, ainsi qu'un estradiot, soldat originaire d'Albanie ou de Grèce. La Mort, elle-même armée d'un court glaive, emmène, en le tenant par le poignet droit, ce dernier, qui porte un yatagan. Contrairement aux autres personnages de la Danse, aux faciès très expressifs, comme le cardinal, les moines, le bourgeois, le guerrier, le jeune noble, le fou, la putain, ces étrangers affichent des mines à peine incrédules ou tristes, mais toujours impassibles. En fait, il s'agit d'une peinture dans la peinture, où les modèles sont figés à la manière de mannequins. Ils ont posé pour le peintre qui, lui, est en plein mouvement, transposant ses croquis sur la toile. Connaissant ses origines, rien d'étonnant que Manuel ait choisi comme sujet pour son dernier tableau des étrangers comme lui, au statut précaire non seulement à Berne, mais dans n'importe quelle ville européenne. Les uns, parce qu'ils sont les premiers à être chassés dès qu'un malheur s'abat sur la communauté dans laquelle ils ont tenté de prendre racines, ou encore pour «purifier» la terre d'accueil, comme le prouvent les exactions contre les Juifs partout en Europe (l'expulsion des Sépharades en 1492, les chantages que les Juifs subiront bientôt dans le seul endroit en Europe qui leur garantissait la sécurité, Venise, jusqu'à la fin du XVIII ${ }^{\mathrm{e}}$ siècle). Les autres, parce qu'ils représentent le danger qui vient de l'Orient, la volonté des Ottomans de conquérir l'Occident et, par là, de menacer la religion chrétienne (leur premier siège de Vienne aura lieu quelques années après l'œuvre de Manuel, en 1529). Dans ce $44^{\mathrm{e}}$ tableau, il s'agit d'un rapprochement entre Manuel et ceux qui ne font pas partie de la communauté bernoise, même si le peintre, dans le texte accompagnateur, prend ses distances eu égard aux intrus. La Mort les mène à leur damnation éternelle ${ }^{9}$.

La représentation du peintre est remarquable à plus d'un point de vue. Manuel se montre dans un portrait en pied, vêtu d'un habit, comme les aimaient les hommes de la Renaissance, plus particulièrement les Reyslouffer suisses. Le terme est une contraction de "Reise» (voyage) et "laufen» (courir), euphémisme désignant les jeunes hommes de la Confédération qui accourent dès qu'une guerre se dessine, pour vendre leurs services au meilleur offrant, même si c'était au risque de devoir s'affronter les uns les autres. Pour la période en question, le but $\mathrm{du}$ voyage était invariablement l'Italie du Nord, endroit stratégique sur l'échiquier des trois grandes puissances européennes: l'Empire, la papauté, ainsi que la France qui tente de briser son encerclement par les Habsbourg. 
Manuel se présente sous le jour du mercenaire classique, coiffé d'un chapeau rouge posé de côté afin de dégager le profil caractéristique au nez aquilin ${ }^{10}$. Le pourpoint aux manches immenses, en brocard vert pistache, est négligemment jeté sur les épaules, par-dessus la chemise plissée. Les chausses sont de couleurs différentes, avec des nœuds assortis sous les genoux: la jambe gauche, en rouge et or, est ornée de nombreux crevés, tandis que le tissu de la droite est bleu et jaune. Ses chaussures noires, légères et confortables, sont des "gueules de vache» (Kuhmäuler), si prisées des Reyslouffer. La jambe gauche est tournée vers le spectateur, la braguette surdimensionnée est également ornée de crevés. Dans la main gauche, Manuel tient la palette et le bâton d'appui. De la droite, il peint le groupe des personnages que la Mort emmène. Devant lui, une table basse sur laquelle sont alignés les outils du peintre, pinceaux et pots de peinture. Le profil de Manuel révèle un sourcil fortement courbé, des lèvres pleines, un menton et une mâchoire volontaires. Les cheveux sont dorés, bouclés.

Derrière le peintre, un squelette est à quatre pattes sur le sol, un sablier sur le dos. Le crâne dirige des yeux vides sur la main de l'artisan. Le bras droit agrippe le bâton pour le lui retirer. Alors que le crâne est peint de manière réaliste, le corps du squelette reprend la manière dont Manuel avait déjà montré la Mort dans un petit tableau daté de 1517, «La Mort et la jeune fille»(Der Tod und das Mädchen $)^{11}$, où le squelette ouvre la bouche pour le dernier baiser tout en relevant de la main gauche la jupe de la jeune fille et cherchant son sexe. Il s'agit plutôt d'un cadavre en décomposition, avec des lambeaux de chair qui pendent de la cage thoraxique avec, çà et là, les restes de l'habillement caractéristique du Reyslouffer.

En arrière-plan s'ouvre l'arche, de goût italien et posé sur des colonnes trapues, donnant la vue sur un paysage idéal, suisse sans doute, avec des montagnes, des champs cultivés, un lac et deux bateaux qui s'approchent d'une ville située sur la rive (ce genre de paysage se retrouve dans presque tous les tableaux de Manuel). En haut, à gauche, au-dessus du groupe des infidèles, sont présentées les armoiries de Bernhard Tillmann ${ }^{12}$, célèbre orfèvre bernois et, avec Manuel, membre du Grand Conseil (les «Deux Cents»), tandis que celles du peintre, trois lys dorés sur fond bleu au-dessus de bandes rouges et or, occupent le coin droit de la scène. L'expression de Manuel est à la fois sérieuse et sereine: c'est le portrait idéal du jeune noble de son temps, élégant, mince, plein de vie. En effet, le Reyslouffen anoblissait un jeune paysan, un artisan, un bourgeois ${ }^{13}$; le mercenariat lui assurait une place de choix dans la société, il lui conférait du prestige.

Mais quand le temps de vie du peintre est écoulé, la Mort s'adresse à lui en ces termes:

[La Mort] :

Manuel, tu as peint tous les personnages

du monde sur ce mur;

maintenant, tu dois mourir,

rien ne t'aidera,

tu n'as de certitude ni quant

à la minute, ni quant à l'heure.

[Manuel]:

Aide-moi, mon seul Sauveur,

je t'en prie,

car je dois partir de ce monde.

Quand la Mort me coupera la parole,

alors [j'espère] que Dieu vous garde,

mes chers compagnons ${ }^{14}$.

(Traduction de l'auteur.)

La Mort reprend l'essentiel d'une phrase que Manuel a inscrite maintes fois dans ses dessins : "Personne ne peut tout savoir» (Nieman kans als wüssen), une indication on ne peut plus claire de la pensée du peintre, demeurée redevable à celle du Moyen Âge. Mais elle indique en même temps l'incertitude qui prévalait en ce début $\mathrm{du} \mathrm{XVI}^{\mathrm{e}}$ siècle, où les alliances politiques changent du jour au lendemain, où la tradition des pratiques religieuses, minées depuis deux siècles par la politique papale, est si fortement ébranlée que le bouleversement de l'ordre social est imminent. Manuel se résigne; de là l'expression détachée, presque sereine, de son autoportrait.

Il faut rappeler également que la villeÉtat avait été secouée par des scandales qui en firent la risée de l'Empire. D'abord, en 1509, les «apparitions » de la Vierge à un frère dominicain, orchestrées par quelques moines (ce qui leur valut de finir sur le bûcher); ensuite, en 1518, l'achat d'une relique convoitée, le crâne de sainte Anne, qui se révéla être un faux. S'ajoutaient à cela la terrible défaite des mercenaires à Marignan lors de la reprise de Milan par les troupes françaises (1515); les procès au sujet de l'interdiction de s'engager comme mercenaires, procès qui impliquèrent bon nombre de dignitaires de la ville; la mort inattendue du bailli Wilhelm von Diesbach; les pratiques scandaleuses de Bernhardin Sanson, vendeur de lettres d'indulgences ${ }^{15}$.

Quand on connaît les événements qui se sont déroulés à Berne entre 1516 et 1519 , il n'est pas surprenant d'en retrouver les effets dans ces portraits de Burger bernois. Cependant, ce n'est qu'en 1742 que la question concernant la présence du peintre a préoccupé l'historien Samuel
Scheurer qui a tenté de l'expliquer dans son ouvrage La vie et les ouvres importantes de Niclaus Manuel (Leben und wichtige Verrichtungen Niclaus Manuels):

Sans doute, Niclaus Manuel a entendu des plaintes selon lesquelles bien des prédicateurs prêchent aux autres, tout en s'oubliant eux-mêmes. Afin

de ne pas commettre cette erreur

commune, il s'est donc rappelé sa propre mortalité, et s'est peint au moment où la Mort l'emmène ${ }^{16}$.

(Traduction de l'auteur.)

Le «sans doute» qui ouvre la réflexion de Scheurer, indique une spéculation de sa part, tout comme je l'ai fait moi-même et d'autres avant moi sur les raisons de la présence du peintre dans sa Danse macabre. Cependant, à la fin de l'ère baroque, les informations au sujet de Manuel étaient encore enfouies dans les archives, et bon nombre de ses tableaux qui avaient échappé aux iconoclastes n'étaient pas repérés. Il faudra attendre les travaux patients, effectués par des chercheurs aux $\mathrm{XIX}^{\mathrm{e}}$ et $\mathrm{XX}^{\mathrm{e}}$ siècles pour resituer le peintre-poète-homme d'État dans son temps. Ce qui me ramène à la question posée au début de cet article, à savoir: pourquoi un peintre suisse a-t-il composé une danse macabre en pleine Renaissance, alors que son exécution est redevable au Moyen Âge tardif? Nous connaissons désormais le comment de la Danse, ainsi qu'une partie de la réponse quant à son existence.

Résumons: j'ai émis l'hypothèse que l'autoreprésentation dans un tableau, accessible en tout temps et vu par un maximum de personnes, devait assurer au peintre des revenus importants ${ }^{17}$. Son portrait sur le mur du cimetière le fait connaître comme peintre et comme poète. Considérant le fait que Manuel était le fils d'un père d'origine étrangère, j'ai cru pouvoir déduire qu'avec sa présence dans un tableau situé de façon à attirer l'attention des passants, juste à la fin de la série, le peintre se positionnait à l'intérieur de la société bernoise - condition sine qua non pour sa future carrière politique.

J'en viens à un point plus délicat. Tous les chercheurs s'accordent pour dire que les années d'apprentissage de Manuel se sont déroulées à Berne, dans un atelier de vitraux, et qu'il fait montre d'une certaine aisance dans le dessin. En fait, mieux que les pinceaux, il manipule le fusain, la pointe d'argent, la plume. Perspicace, doté d'un esprit qui saisit avec justesse, et rapidement, les événements de son temps, il sait qu'il n'atteindra jamais le niveau d'un Dürer, d'un Hans Holbein ou de n'importe quel grand maître de son temps, à la réputation internationale. Il ne pourra pas faire carrière auprès d'une grande cour, 
LA VIOLENCE DES PROPOS, LE MANQUE DE RESPECT ENVERS LES DIGNITAIRES, LE LANGAGE CRU, TOUT TRADUIT LE DÉSENCHANTEMENT, LE DÉGOÛT, L'EXTRÊME IMPATIENCE DU FIDÈLE ENVERS UNE INSTITUTION QUI, À CAUSE DES SCANDALES À RÉPÉTITION, AVAIT PERDU SA CRÉDIBILITÉ.

comme Holbein à celle d'Henri VIII, en Angleterre. Alors, se conformant à l'esprit de la Réforme, il offre ses services à sa ville natale. Ce n'est pas un hasard s'il envoie, lors d'une expédition en Italie, en 1522, une lettre en ce sens au gouvernement bernois et s'il écrit, dans la même année, une chanson devenue célèbre partout dans la Confédération après la bataille de Bicocca. Dès ce moment, il abandonne la peinture et ne revient que sporadiquement au dessin (un des rares de cette période que nous connaissions, Le Christ et la femme adultère, date de 1527; un an avant sa mort, Manuel a signé une dernière représentation d'un mercenaire suisse, où se note un maniérisme certain dans la facture).

Bref, Manuel est un bon artisan qui n'a pas eu l'occasion de développer pleinement son talent d'artiste: les réponses des personnages de la Danse à la Mort sont simples et directes. Les dignitaires de l'Église tout comme les Burger sont peints rapidement (c'est la technique al fresco qui en est responsable, bien qu'on discute encore si certains panneaux n'ont pas été peints à l'huile [?]), assez crûment, de manière à être compris et interprétés sans ambiguité tant par le plus humble des fidèles que par les commanditaires. D'un autre côté, certains tableaux, pour la plupart des commandes de Burger, et des retables qui nous sont parvenus, témoignent de l'immense potentiel artistique de Manuel, potentiel qui se situait surtout dans l'audace de sa pensée. Je rappelle simplement qu'un Tüchle ${ }^{18}$ comme Le Jugement de Pâris $(1517 / 1518)$ met en scène le berger troyen dans une attitude unique parmi les quatre cents traitements répertoriés du sujet en peinture: au lieu de se laisser plonger par Mercure dans un rêve, car un mortel ne supporterait pas la lumière dégagée par les déesses, Pâris dirige son regard directement dans les yeux de Vénus qui vient de recevoir la pomme, destinée «à la plus belle », tandis que Junon et Pallas sont en train de comploter pour la perte de Troie et la mort du fils de Priam. Ici, par l'échange des regards, Manuel fait preuve d'une assurance exceptionnelle quant à l'interprétation du mythe. En même temps, les erreurs techniques sautent aux yeux : anatomie un tant soit peu incertaine, surtout pour les pieds, plus particulièrement ceux de Vénus et de Pâris, utilisation fautive de pigments qui ont détruit une partie de la toile, etc., même si nous savons que d'autres que lui ont par la suite retouché le tableau, à plusieurs reprises. Mais devant l'ensemble de cette œuvre, les manquements perdent de leur importance. Il reste le génie du peintre qui a repensé et modernisé le mythe, établissant le lien entre la peinture conventionnelle qui était à la mode au nord des Alpes et le regard, d'égal à égal, du mortel de la Renaissance sur la divinité. Ailleurs, par exemple dans le retable décrivant le Martyre des dix mille chevaliers, les figures de saint Achatius et de sainte Barbe fournissent la preuve que le métier de Manuel se comparait avantageusement à celui de n'importe quel peintre établi dans une des villes d'importance moyenne de l'Empire germanique.

Par sa Danse macabre, Niklaus Manuel établit un trait d'union entre le passé et les temps mouvementés qui s'annoncent. À ma connaissance, c'est un des rares peintres hors d'Italie dont la pensée ait su garder ses racines dans le Moyen Âge tardif, sachant en même temps s'adapter et, pourquoi pas, tirer profit de la nouvelle ère. Dans son autoportrait, il accepte sa mort. Pour le moment, il est peintre encore, nullement effrayé et encore moins dérangé par le squelette qui l'arrête dans son travail. Mais voilà : les portraits de ses étrangers, Turcs et Juifs, ont été achevés avant que la Mort n'intervienne. Tout compte fait, audelà de l'arche, la vie continue. Avec elle, le Temps avance, le Temps qui se mesure dans un échange éternel entre la Vie et la Mort.

\section{Bibliographie}

HEINZ, M. (1979). "Zum Thema "Niklaus Manuel und die Glasmalerei" », dans Niklaus Manuel Deutsch. Maler, Dichter, Staatsmann, Berne, Kunstmuseum.

KUTHY, S. (1999). Niklaus Manuel im Kunstmuseum Bern, Berne Kunstmuseum.

SCHEURER, S. (1742). «La vie et les œuvres importantes de Niclaus Manuel», dans Bernisches Mausoleum, II, Berne.
TRIPPS, J. (2005). «Den Würmern wirst Du Wildbret sein", Der Berner Totentanz des Niklaus Manuel Deutsch in den Aquarellkopien von Albrecht Kauw (1649). Bern, Verlag Bernisches Historisches Museum, Band 6.

VALERIUS, A. (1884-1901). Die Berner Chronik des Valerius Anshelm. Berne, Wyss, Historischer Verein des Kantons Bern (éd.). 6 vol.

VON TAVEL, H.C. (1991). «Zeichen der Freiheit ", Ausstellung des Europarates 1991, 21, Berne, Bernisches Historisches Museum und Kunstmuseum.

VON TAVEL, H.C. (1979a), Niklaus Manuel. Zur Kunst eines Eidgenossen der Dürerzeit. Berne, Verlag K.J. Wyss Erben.

VON TAVEL, H.C. (1979b). « Niklaus Manuel als Maler und Zeichner", Niklaus Manuel Deutsch. Maler, Dichter, Staatsmann, Berne, Kunstmuseum.

\section{Notes}

1. Le terme désigne des gravures sur bois, réunies sous forme de livre, et habituellement accompagnées de courts textes. En général, gravure et texte se trouvaient sur un même bloc de bois (d'où le nom), et ont été produits surtout en Allemagne et dans les Pays-Bas, au milieu du XV siècle. Des 33 œuvres connues, l'Ars moriendi et la Biblia pauperum sont les plus célèbres.

2. En fait, il s'agit de 24 panneaux doubles, dont deux ne comptent qu'une seule scène, comme le treizième, commandé par l'ordre des chevaliers Teutoniques de Köniz et son chef, Rudolf von Fridingen; sur le dernier, le $46^{\mathrm{e}}$, sans armoiries, un prédicateur parle à une foule de cadavres et de mourants, étendus sous un arbre ou suspendus dans les branches, les uns transpercés de flèches, les autres attendant la faux de la Mort qui, dans l'habit d'un voyageur, enjambe le corps d'un enfant. Dans son commentaire de la scène 45 , J. Tripps mentionne d'autres exemples de l'artisan qui fait partie de la danse, comme Hans Hug Kluber (1535/36-1578) lors de la restauration de la danse macabre de Bâle (1568), ou encore celle, perdue, de Kientzheim, en Alsace. Voir Tripps, 2005, p. 98.

3. Hans Christoph von Tavel, éminent expert de l'œuvre manuelienne, est d'avis que l'ensemble s'étendait sur plus de 100 mètres (voir Zeichen der Freiheit), tandis que J. Tripps l'estime à 80 mètres. Je m'appuie sur la copie de la Danse, exécutée par Albrecht Kauw (1621-1681), qui est plus ancienne que celle de Wilhelm Stettler (1643-1708), reproduite dans l'ouvrage de Kuthy (1999, en noir et blanc, avec des reproductions fortement réduites). Les deux peintres ont connu l'original de la Danse avant sa destruction en 1660, quand le mur du cimetière fut abattu sur ordre du gouvernement de la ville, «afin d'élargir la rue [de l'Arsenal]». Malgré les rénovations de 1554, à ce moment, les fresques étaient sans doute très endommagées, les pigments se détachant ou se dégradant à cause des intempéries. Voir Tripps, 2005, p. 17. 
4. Voir Heinz, 1979, p. 67-73.

5. Le statut des Dürer, Michel-Ange ou Léonard de Vinci était très différent, bien entendu: papes, empereurs et rois honoraient le génie de ces peintres en les invitant à leur table. Dans une lettre, Dürer énumère la liste des honneurs reçus lors de sa visite à Venise et s'émerveille du respect que son art inspire aux Italiens.

6. Voir Hans Christoph von Tavel, dans son essai Niklaus Manuel. Zur Kunst eines Eidgenossen der Dürerzeit, 1979a, p. 99.

7. Puisqu'il s'agit d'une copie du texte original, la graphie de la citation est incertaine. Cependant, on peut supposer que le sens et l'essentiel des quatrains de Manuel n'ont pas subi de changements majeurs. Avant même la Réforme, le peintre exprime clairement les doléances des fidèles. Ce ne sera que le 28 janvier 1528, après une disputatio à Berne qui donne la victoire à la Réforme, que la fureur iconoclaste se déchaînera: en une seule journée, les 25 autels de la cathédrale Saint-Vincent seront détruits, les retables arrachés et brûlés.

[La mort]

Herr Apt, Jr sind gar grosz und fë̈ß/ springend mit mir an disen krë̈ß/ Wie schwÿtzend Jr so kalten schweÿß/ pfuch, pfuch, Jr lond ein großen scheÿß/

À quoi le prieur répond:

Die schläckli hannd mir so wol gethan/ groß guot han Jch Jnn hennden ghan/

Zuo mins Lÿbs wollust han Jchs gwendt

Min Lÿb wirt Jetz von Würmen geschendt

8 Dès 1523, Manuel change de métier: il se consacre de plus en plus à la littérature pamphlétaire, avec deux «jeux carnavalesques ", dont la représentation se fit à Berne, rue de la Croix (la Kreuzgasse), titrés «Du pape et de son clergé» (Vom Papst und seiner Priesterschaft) et "De l'opposition entre le pape et le Christ» (Von Papsts und Christi Gegensatz). Par ces prises de position, Manuel s'identifie clairement à la Réforme et sait s'attirer beaucoup de sympathies dans la société bernoise. La même année, il obtient le poste de bailli (Landvogt) au château d'Erlach, important poste administratif. En 1525, il rédige une autre pièce au titre évocateur, "Le marchand d'indulgences» (Der Ablasskrämer) et, un an plus tard, des dialogues traitant de la Réforme, «Le voyage à Bade d'Eck et de Faber » (Ecks und Fabers Badenfahrt, mettant en scène le célèbre Dr Eck, opposant de Luther), ainsi que Barbali.

9. [La Mort]

Jr Juden Jr unglöübigen Hünd/ köndtend Jr noch souil lÿst vnd fünd/ mueßent Jr dennocht stärben Jn Eewigkheÿt/ Dann Jr hannd verlougnet die Christenheÿt.

Vous, les Juifs, chiens infidèles,

Même avec toutes vos ruses et vos talents, Vous devez mourir pour l'éternité,

Car vous avez nié la chrétienté.

À quoi les Juifs répondent:

O wie sind wir so gantz betrogen/

Die Rabinen hannd vunns alls erlogen/

Sÿ gabenn vnns vil falscher gsatz/
Der tod fuert vnns of helschen platz/

Ô combien on s'est joué de nous,

Les rabbins ont inventé tous ces mensonges, Ils nous ont donné rien que de faux enseignements,

La Mort nous emmène en enfer.

(Texte selon la copie de Kauw; traduction de l'auteur.)

Comme le mentionne J. Tripps, les Juifs devaient porter depuis 1215 un chapeau pointu, de couleur jaune, depuis 1229 le cafetan avec un anneau ou une petite roue jaunes. Depuis l'expulsion des Juifs de Berne (1427), on y recevait rarement des visiteurs de religion juive, pour la plupart des médecins ou des chirurgiens. Voir Tripps, 2005, p. 98.

10. On connaît un autre autoportrait, celui du Musée des Beaux-arts de Berne ( $\mathrm{n}^{\circ}$ d'inv. 326), signé NMD, avec dague suivie d'une boucle, daté de 1520 , technique mixte sur parchemin. L'œuvre présente le peintre non pas de profil, mais en «trois-quart». Le chapeau et la chemise sont presque identiques à ceux de la fresque, bien que les traits soient plus charnus. Cependant, je ne doute pas que le Pâris du Jugement soit également un autoportrait, avec la différence que les cheveux sont d'un châtain clair au lieu d'être blonds, mais le profil est le même. Il se peut que le personnage se trouvant entre le bourreau et Salomé, dans la première version $(1513 / 1514)$ de la décapitation de saint Jean, un jeune homme au visage «tordu de douleur» qui regarde le spectateur, soit également un autoportrait (H. C. von Tavel, 1979b, p. 331).

11. Aujourd'hui au Musée de Bâle. Il se trouve sur l'envers de «Bethsabée au bain» (Bathseba im Bade).

12.Tillmann et Manuel participeront activement au "nettoyage» de la cathédrale Saint-Vincent, en 1528. De l'orfèvre, seuls quelques magnifiques spécimens de son travail, des commandes exécutées pour des Burger, ont survécu. Cependant, tout le trésor de la cathédrale (reliques, objets sacerdotaux, calices, un masque célèbre en or) sera fondu et transformé en monnaie.

13. Après une bataille où ils s'étaient distingués par leur bravoure, bien des mercenaires reçurent le titre de "chevalier», que ce soit du pape, du roi de France ou encore de l'empereur, comme lors de la prise de la forteresse de Gênes par les Français (1507), la guerre devant Milan, contre la domination française, et la restitution de cette ville à Maximilien Sforza (1512), la victoire des Suisses lors de la bataille de Novare, contre les Français (1513). En 1516 , certaines villes confédérées, comme Berne, Fribourg, Lucerne, acceptent une «paix éternelle» avec la France. La même année, des Reyslouffer se retrouvent dans des camps opposés (François I ${ }^{\mathrm{er}}$ contre l'empereur Maximilien $\mathrm{I}^{\mathrm{er}}$ ). Chaque bataille fut menée avec une férocité rare; les lansquenets, mercenaires impériaux, redoutaient les Suisses à cause de leur violence dans le combat. L'intérêt des Reyslouffer était double: le gain avant tout, puisqu'ils pillaient le camp ennemi et spoliaient les morts, et les pen- sions reçues des chefs de guerre, ensuite le prestige des titres de noblesse.

14. [La Mort]

Manuel aller Wällt Figur/

hast du gemalet an dise Mur/

Nun muost stärbenn da hilfft kein Fund/

Bist ouch nit sicher Minut noch Stund/

Et la réponse de Manuel:

Hilff einiger Heÿland, drumb ich dich bitt/

Dann hie ist gar keines blÿbens nit/

So mir der tod min Red wirt stellenn

So bhuet üch Gott, mine lieben Gsellen

15. Valerius Anshelm, dans sa chronique officielle de Berne, relate qu'en 1518, Bartlomé May, un membre du Petit Conseil, dut s'agenouiller devant Sanson et répudier Luther. On avait trouvé chez May un exemplaire des thèses luthériennes. Ailleurs, Anshelm raconte qu'un jeune patricien, Jakob vom Stein, avait cédé à Sanson sa monture préférée, un superbe étalon gris, contre une indulgence complète pour lui-même ainsi que pour cinq cents mercenaires suisses sous son commandement, sans oublier tous ses ancêtres et l'ensemble de ses serfs au village de Belp. Voir Die Berner Chronik des Valerius Anshelm, 6 vol.

16. "Ohne zweifel hatte Niclaus Manuel mehrmals Klägten gehört führen, dass viele Prediger wohl anderen predigen, sich selbst aber darbey vergessen. Derowegen, nicht auch in diesen allzugemeinen Fehler zu verfallen, predigte er sich selbst die Sterblichkeit auch, und mahlete sich als vom Tod hingerissen.» (Samuel Scheurer, La vie et les cuvres importantes de Niclaus Manuel, cité d'après Sandor Kuthy, 1999, p. 61.)

17. La production manuélienne, dessins et tableaux confondus, s'étend de 1507 à $1521 / 1522$, avec une forte concentration dans les années 1513 à 1520.

18. Le terme signifie, littéralement, "chiffon». Dans le cas du Jugement, il s'agit d'une commande, exécutée rapidement sur une toile de lin cru, imitant une tapisserie. Au XVI ${ }^{\mathrm{e}}$ siècle, ce genre de peinture était très répandu; à cause de leur extrême fragilité, peu de ces Tüchle ont pu être sauvés. 